\title{
Fiberbundle Receiver: A new Concept for High-Speed and High-Sensitivity Tracking in Optical Transceivers
}

\author{
Christian Fuchs ${ }^{a} *$, Hennes Henniger ${ }^{a}$, Dirk Giggenbach ${ }^{a}$ \\ ${ }^{a}$ German Aerospace Center (DLR), Muenchner Str. 20, 82234 Wessling, Germany
}

\begin{abstract}
The design of optical transceivers for mobile free space optical communication systems is a sophisticated task. A typical transceiver design includes the combination of coarse- and fine-tracking-sensors, a data receiver, and a laser source for sending data. For heavily vibrating environments or for systems that have stringent requirements in terms of pointing accuracy, a fast steering mirror might be used. This results in a design with many parts.

The Fiberbundle Receiver, consisting of 7 fibers in conjunction with photodiodes, can ease such an implementation process. It allows the combination of some of the mentioned components, which results in a less complicated design. Standard COTS components for fiber based WDM systems can be used for challenging tasks as e.g. wavelength splitting. Furthermore, a cost-effective and very fast tracking system can be implemented. A technology demonstrator for the application of the Fiberbundle Receiver at a wavelength of $1550 \mathrm{~nm}$ has been developed and will be discussed in this paper.
\end{abstract}

Keywords: Optical Transceiver, Fiberbundle, Fast Tracking, High Sensitivity

\section{INTRODUCTION}

Free space optical (FSO) communication links seem to be one solution for the increasing demand of datarate in mobile applications. The maturity of mobile FSO links has recently been demonstrated in a number of projects, e.g. in the FASOLT experiment, ${ }^{1}$ the CAPANINA High-Altitude-Platform (HAP) Downlink ${ }^{2}$ or the optical downlink from the japanese satellite KIRARI to Oberpfaffenhofen, Germany. ${ }^{3}$ Astrium, Toulouse, demonstrated in the LOLA experiment a link between an aircraft and ESA's geostationary telecoms test satellite ARTEMIS, with several successful trials in December 2006. Further fields of research include the development of mobile ad-hoc networks (MANETs) in aeronautical environments by utilizing FSO links. ${ }^{4,5}$

In order to bring mobile FSO technology to the market, reliable and reasonably prized optical communication terminals are required. However, the implementation of full-duplex optical transceivers - as necessary for most of the described applications - is a challenging task. A Fiberbundle Receiver, as it is described in the scope of this paper, eases several of the typical design challenges.

\section{THE CONCEPT OF THE FIBERBUNDLE RECEIVER}

A typical optical transceiver as shown in Figure 1 is composed of many different components. This includes a Coarse Tracking Sensor (CTS, e.g. an InGaAs camera for a wavelength of $1550 \mathrm{~nm}$ ), a Fine Tracking Mechanism (FTM, e.g. a fast steering mirror), a laser source for data transmission and finally a data receiver in conjunction with a Fine Tracking Sensor (FTS, e.g. a 4 quadrant detector).

It seems important to mention that especially the requirements for the chromatic mirrors, that are used for the separation between $\mathrm{Rx}$ - and Tx-wavelengths, are very stringent, in order to avoid any reflections from the Tx-laser to the data receiver. The complexity of this wavelength splitting increases if the used wavelengths are close together, as it is the case for eye-safe communication systems that operate in the range of $1550 \mathrm{~nm}$, and is not possible with COTS components. Custom-built components that are capable of fulfilling the separation requirements are expensive and difficult to obtain.

\footnotetext{
${ }^{*}$ Christian Fuchs; E-mail: christian.fuchs@dlr.de; Telephone: +49 (0)8153 28-1547
} 


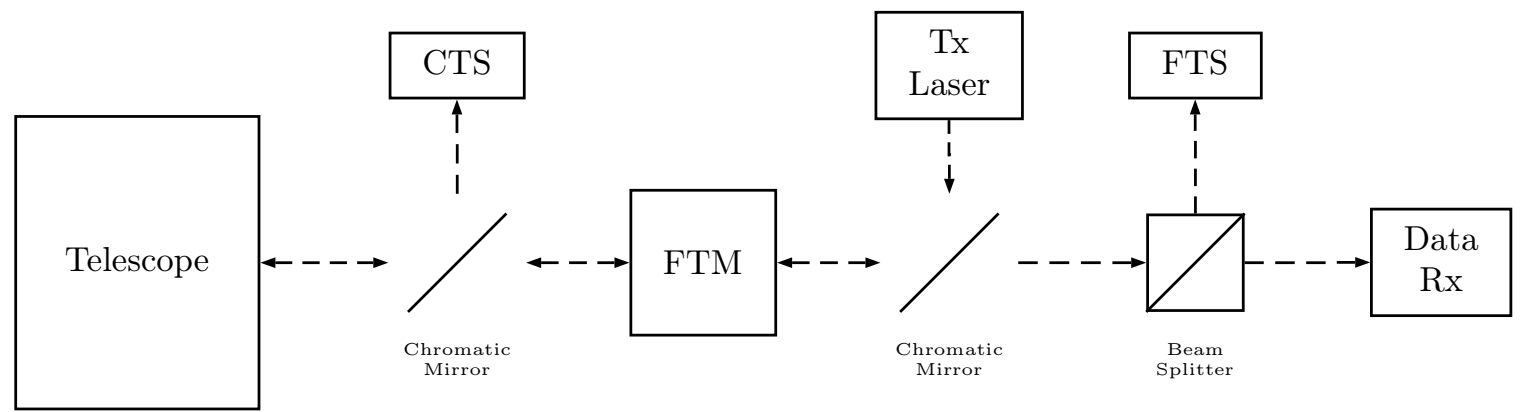

Figure 1. Example Block Diagram of a conventional Optical Transceiver

The fiber-bundle concept allows to integrate all parts behind the FTM, as can be seen in Figure 2. The 6 outer fibers are fed to the tracking system, while the central fiber supplies the data receiver. A Y-Coupler allows to couple light from the Tx-laser into the central fiber, i.e. it is used simultaneously for sending and receiving data. For the purpose of wavelength separation, standard parts that were developed for fiber-based WDM communication systems can be used. The overall number of components is reduced, and only one chromatic mirror is necessary. Furthermore, the mechanical construction of the optical setup is simplified, since many fiber based components can be used and thus alignment requirements drop out.

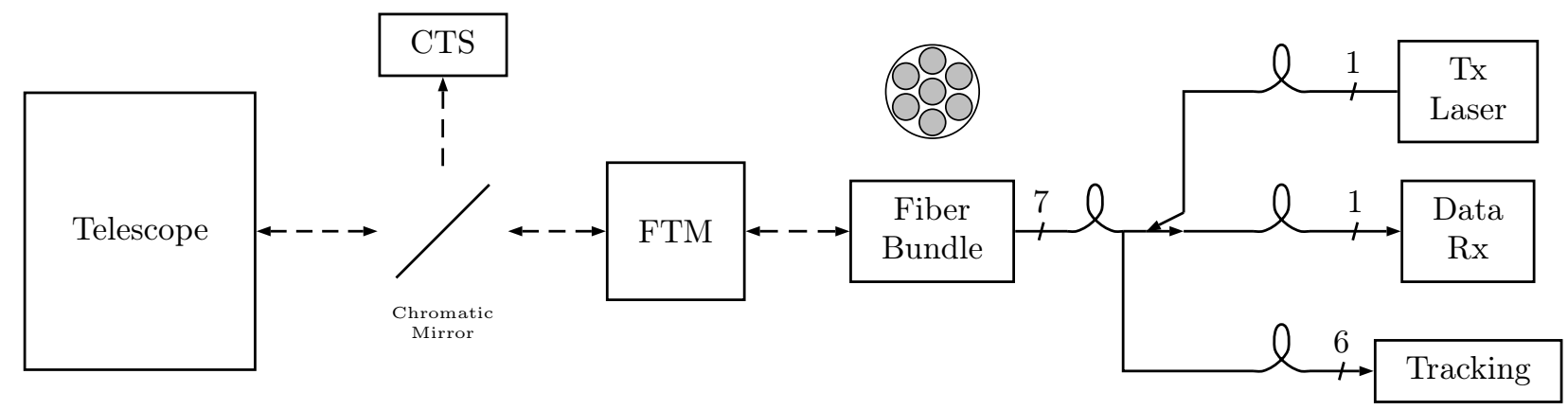

Figure 2. Block Diagram of an Optical Transceiver with Fiberbundle

The applicability of the fiber bundle as fast tracking device will be investigated in the scope of this paper. A block diagram of the realized setup is visible in Figure 3. A fast steering mirror system is placed inside the optical path of a lens in order to reflect the focused incident laser beam to the fiber-bundle. Each of the bundle's seven fibers leads to photodetectors and a signal conditioning electronics, in order to perform the opto-electronical conversion and some filtering of the signals. The PAT Control Unit (PCU) calculates a control signal depending on the power distribution in the 7 fibers. Furthermore, the PCU applies a control loop and controls the mirror. The control unit between PCU and mirror generates a high-voltage signal which is necessary for the mirror system. In order to get an eye-safe system, the wavelength of $1550 \mathrm{~nm}$ was used.

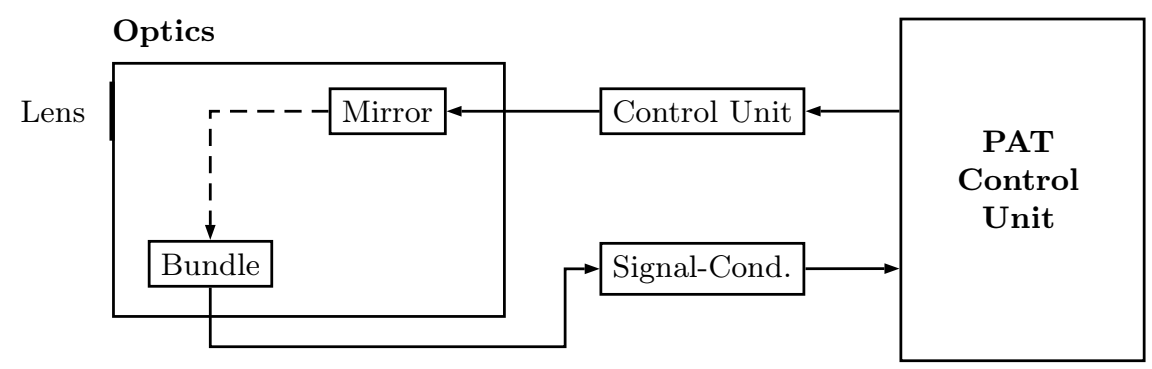

Figure 3. Block Diagram of the Experimental Setup 


\section{TEST BED BUILDING}

\subsection{Fiberbundle}

The used Fiberbundle consists of standard multimode fibers and was custom manufactured. The diameter of each fiber core is $100 \mu \mathrm{m}$, the overall diameter of one fiber (incl. cladding) is $110 \mu \mathrm{m}$. Thus, the total diameter of the bundle is $330 \mu \mathrm{m}$ (with cladding) resp. $320 \mu \mathrm{m}$ (without cladding). A sketch as well as a photography of the Fiberbundle can be seen in Figure 4.
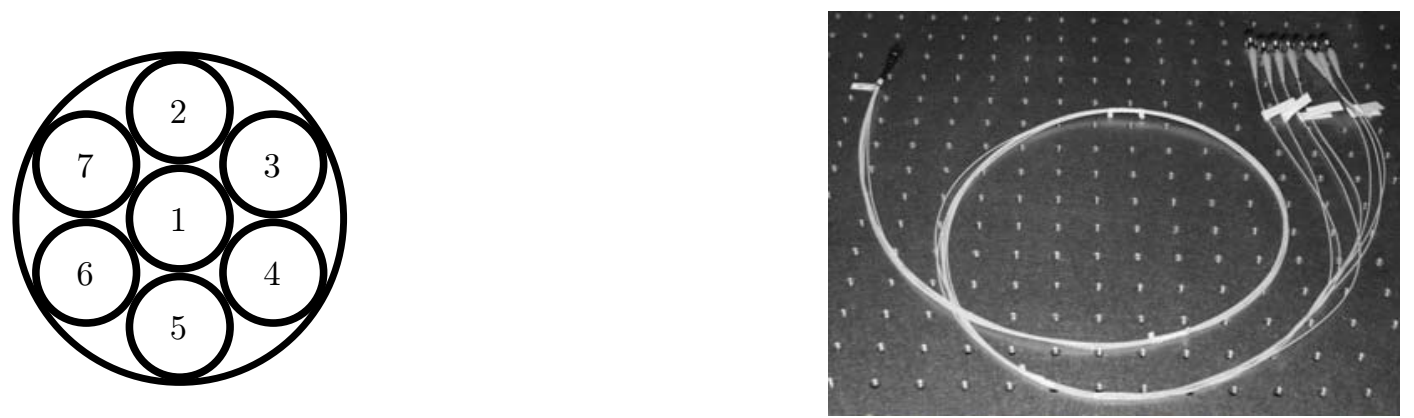

Figure 4. Sketch and Photography of the Fiberbundle

The bundle is mounted in a standard FC connector, while the individual fibers have FC/PC connectors. This allows an easy connection to fiber-coupled photodiodes.

Due to the multimode light propagation inside the fibers, the datarate of the system is limited because of multimode dispersion. The theoretically achievable bandwidth with a typical Bandwidth-Length-Product for a $100 \mu \mathrm{m}$ multimode-fiber of $\approx 10 \mathrm{MHz} \cdot \mathrm{km}$ can be found to be $10 \mathrm{GHz}$ for a fiber length of $1 \mathrm{~m}$. However, since the setup of the Fiberbundle Receiver is not optimized for fiber coupling and the signal coupled into the fiber was propagated through the atmosphere, only suboptimal coupling conditions exist. Therefore, the achievable bandwidth was found to be 1 to 2 orders of magnitude below the theoretical value.

\subsection{Fast Steering Mirror}

A piezo-electrical mirror system has been chosen as beam steering device for the experimental setup. It was manufactured by Marco GmbH, Dachau, Germany and features the characteristics as stated in Table 1.

\begin{tabular}{lc}
\hline \multicolumn{1}{c}{ Parameter } & Value \\
\hline Mirror-Diameter & $25.4 \mathrm{~mm}$ \\
$f_{\text {Res }} x$ & $0.65 \mathrm{kHz}$ \\
$f_{\text {Res }} y$ & $0.65 \mathrm{kHz}$ \\
max. Angle $x$ & $\pm 4.0 \circ^{\circ}$ \\
max. Angle $y$ & $\pm 4.125^{\circ}$ \\
\hline 1. Characteristics of the Mirror System
\end{tabular}

The relatively high resonant frequency of $650 \mathrm{~Hz}$ allows a very fast beam steering, while the big angle of movement enables a huge Field of Regard for the system.

Furthermore, the mirror system is equipped with a position measurement system, that outputs a voltage which is proportional to the current mirror position. This signal will be used for measurement purposes.

\subsection{Optical Setup}

Figure 5 presents a sketch of the optical part for the experimental setup. The used lens is an achromat with a diameter of $75 \mathrm{~mm}$ and a focal length of $300 \mathrm{~mm}$. The lens was designed for visible wavelengths and is thus operated outside its specifications. Since the experimental setup is not operated close to the diffraction limit, it can be expected that this fact has no influence on the operability of the system. The distance $a$, i.e. the mounting 


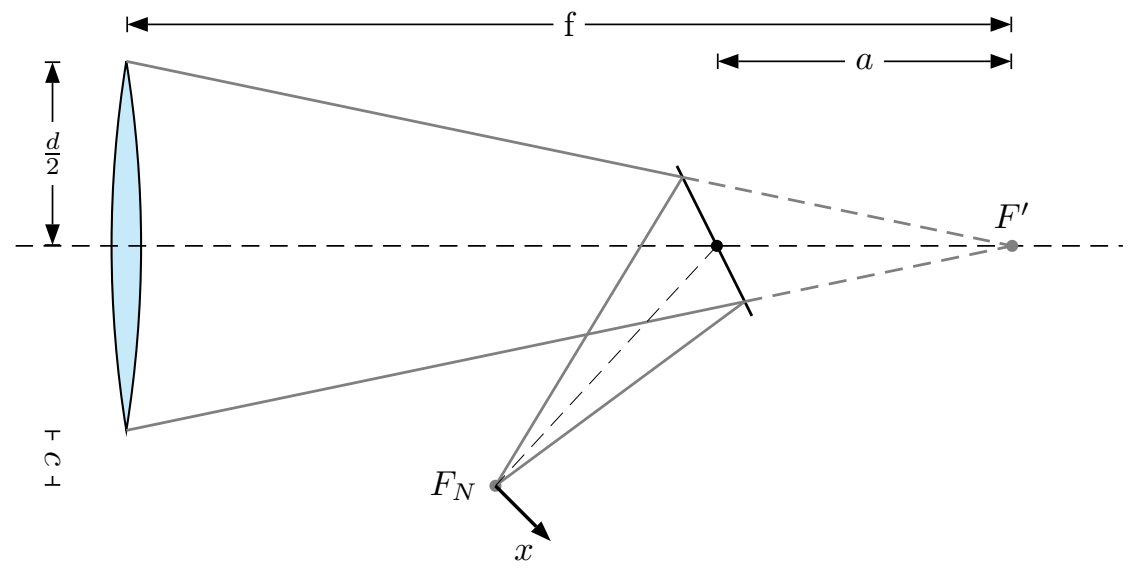

Figure 5. Sketch of the Optical Setup

point of the mirror, has been chosen in such a way that the full aperture of the mirror is used by the converging beam.

Under normal operating conditions, the lens focuses the incoming laser beam onto the central fiber of the bundle and creates a spot on the bundle's surface. If the angle of the incoming beam changes, the spot on the bundle moves from the central to an outer fiber. This condition gets identified by the PAT Control Unit, which generates a control signal for the mirror in order to steer the spot back to the central fiber.

Taking the focal length of the lens $(300 \mathrm{~mm})$ and the total diameter of the bundle $(320 \mu \mathrm{m})$ into account, the Field of View (FoV) of the system can be found to be around $1 \mathrm{mrad}$. The focusing angle of the lens $(\arctan (d / f))$ is well below the acceptance angle of a multimode fiber, i.e. no power is lost for that reason. The Field of Regard (FoR) depends mainly on the mirror's angle range and is with the given values (cp. Table 1) in the order of $85 \mathrm{mrad}$.

A photography of the optical setup can be found in Figure 7.

\subsection{Electronical Setup}

A block diagram of the electronical set-up is shown in Figure 6. The photodetectors are from Thorlabs, type $P D A$ 400. These are InGaAs-PIN-Detectors with a peak response of $0.95 \mathrm{~A} / \mathrm{W}$ at $1550 \mathrm{~nm}$. The following low-passes serve as noise-suppression- and anti-aliasing-filters for the subsequent A/D-Conversion.

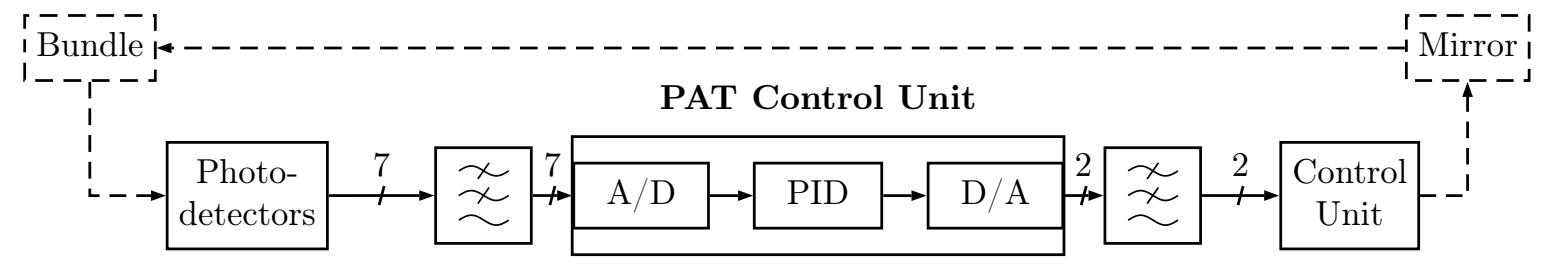

Figure 6. Block Diagram of the Electronical Setup

A Standard PC with a data acquisition card serves as PAT Control Unit (PCU). The data acquisition card is used to sample the signals of the photodetectors at a rate of $1 \mathrm{kHz}$, which is the limit of the card. Afterwards, the data stream is fed to a program that does a spot position calculation. More details about the position calculations will be given in Section 4.3. The program also applies a closed PID control loop to the data. 
Finally, the mirror's control signal is created by converting the output of the PID-filter to an analog voltage. The additional low-passes act as reconstruction filters and the Control Unit creates a high voltage signal, which is necessary to drive the mirror system.

Figure 7 shows a picture of the electronical setup.
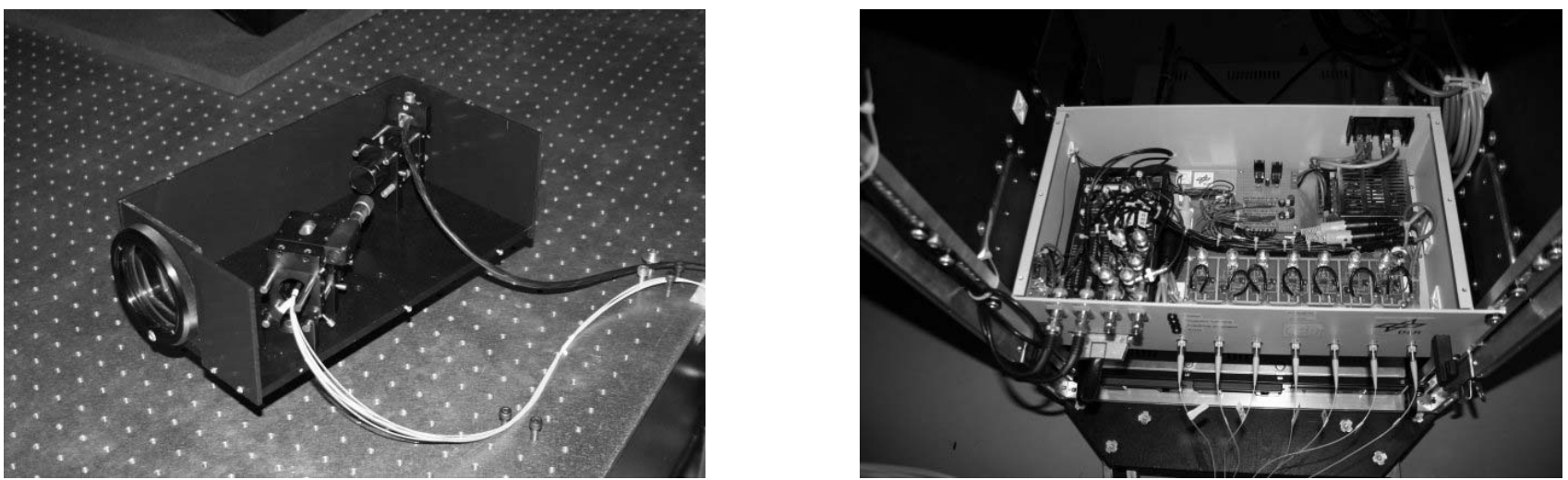

Figure 7. Photographies of the Optical and Electronical Setup

\section{SIMULATION OF THE SYSTEM}

\subsection{Estimation of Spot Position on Fiberbundle}

In order to generate a control signal for the fast steering mirror, an estimate of the spot position on the Fiberbundle needs to be calculated. This can be done by weighing each fiber's position with the power that is currently impinging in the fiber:

$$
\begin{aligned}
x^{*} & =\frac{\sum_{i=2}^{7} P_{i} \cdot x_{i}}{\sum_{i=1}^{7} P_{i}} \\
y^{*} & =\frac{\sum_{i=2}^{7} P_{i} \cdot y_{i}}{\sum_{i=1}^{7} P_{i}}
\end{aligned}
$$

with

$$
\begin{array}{ccl}
x^{*}, y^{*} & {[1]} & \text { Position estimate of spot on Fiberbundle's surface } \\
x_{i}, y_{i} & {[1]} & \text { Position of the corresponding fiber relative to central fiber } \\
P_{i} & {[\mathrm{~W}]} & \begin{array}{l}
\text { Measured power in the corresponding fiber } \\
i
\end{array} \\
\begin{array}{c}
\text { Index of the fiber (cp. Figure 4) }
\end{array}
\end{array}
$$

The coordinate system for this calculations is centered on the central fiber. Since only the relative positions of the vectors $\left(x_{i}, y_{i}\right)$ corresponding to the central fiber are important, no unit is necessary for this parameters. Furthermore, a normalization to the total impinging power in all fibers is accomplished.

Due to the facts that some power gets lost in the gaps between the fibers and that the power in the outer fibers does not increase linearly with the spot offset, this calculations are erroneous. It will be shown later that this non-linearity can be neglected for small offsets from the center.

\subsection{Power Distributions at the Fiberbundle for different distances after the focusing lens}

For the purpose of determining the optimal installation position of the Fiberbundle, the system was simulated in the optical design program Zemax. The power distributions (i.e. spots) for different distances after the focusing lens were recorded and imported into Matlab. A plane wavefront at the lens - as it is the case for a big distance between sending laser and receiver - was simulated. This allowed the usage of realistic power distributions for the simulations. 
The power distributions for the relevant distances close to the lens' focal length are shown in Figure 8, while the FWHM-diameters of the spots are given in Table 2. It is remarkable that the focal length was not found to be exactly the datasheet value of $300 \mathrm{~mm}$. The reason for this is probably the fact, that the lens is not operated in its design wavelength range.

Initially it seems to be desirable to setup the Fiberbundle in such a way that the power in the central fiber is maximized, i.e. with a distance of $296.0 \mathrm{~mm}$. However, this would lead to the condition that power is not constantly impinging in the outer fibers, and no meaningful spot position estimation would be possible with Equations 1 and 2.
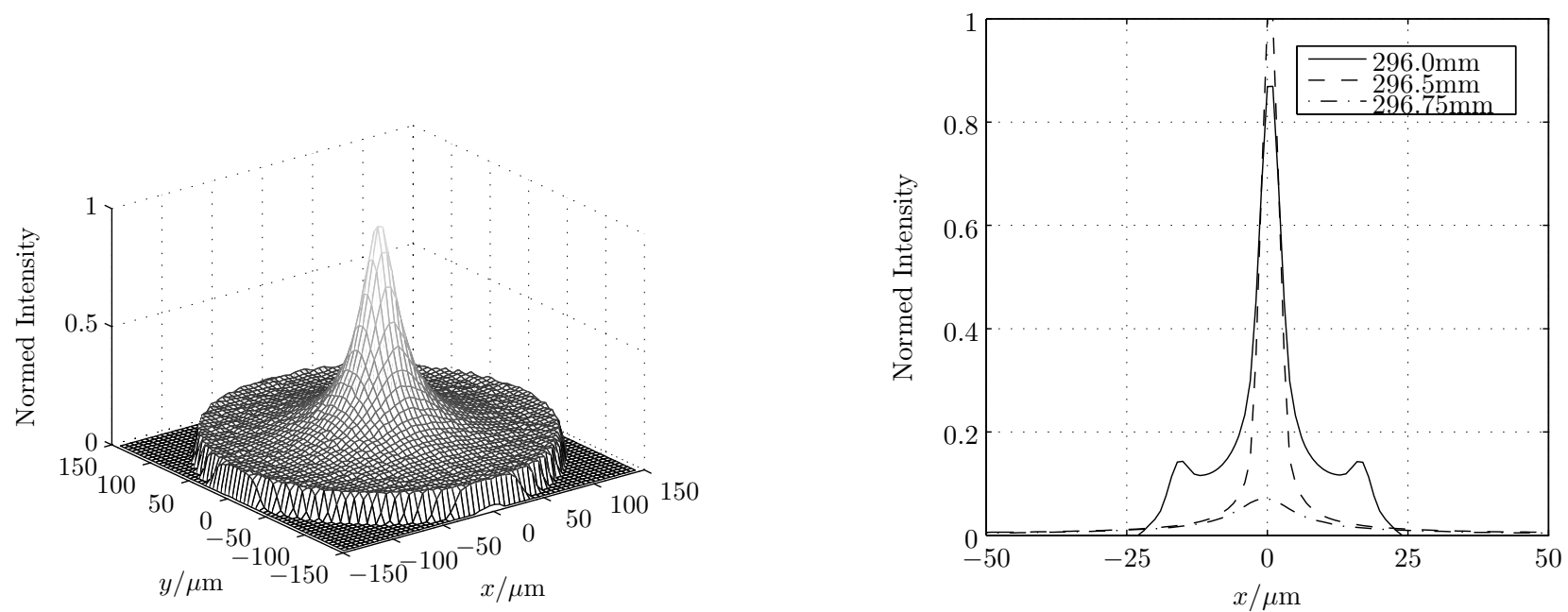

Figure 8. Power Distributions for different installation positions of the Fiberbundle after the focusing lens Left: Example of full power distribution for a distance of $297.0 \mathrm{~mm}$, Right: Cuts through full power distributions $(y=0)$ for different distances

\begin{tabular}{cc}
\hline Distance $/ \mathrm{mm}$ & FWHM-Diameter $/ \mu \mathrm{m}$ \\
\hline 296.0 & 25.5 \\
296.5 & 78.5 \\
296.75 & 122.5
\end{tabular}

Table 2. Spot FWHM-Diameters for different installation positions of the Fiberbundle

Therefore, a greater distance with a bigger spot diameter should be used in order to optimize the behaviour of the controlling system. The power loss that is associated with this adjustment is e.g. with an FWHM-diameter of $75.5 \mu \mathrm{m}$ less than $3 \mathrm{~dB}$ and therewith not greater as for a traditional optical transceiver, where some light is coupled out for the tracking sensor with a beam splitter. The determination of the distance used in this experimental setup will be described later on.

\subsection{Modeling of the Fiberbundle}

The Fiberbundle was simulated numerically in Matlab. Therefore a matrix containing a circle, that stands for one fiber, was created. By cross-correlating this circle with the spots described in Section 4.2, the power in one fiber for different spot positions can be found. An example for the result of this operation is displayed in Figure 9. By applying the position offsets of the outer fibers corresponding to the central fiber, the powers in all fibers are available for a specific spot and its position.

The resolution for the simulation was chosen to $1 \mu \mathrm{m}$. This is well below the diameter of a single fiber $(100 \mu \mathrm{m})$ and the gap between the fibers $(20 \mu \mathrm{m})$. Therefore the simulation results can be expected to be feasible and applicable to the real system. 

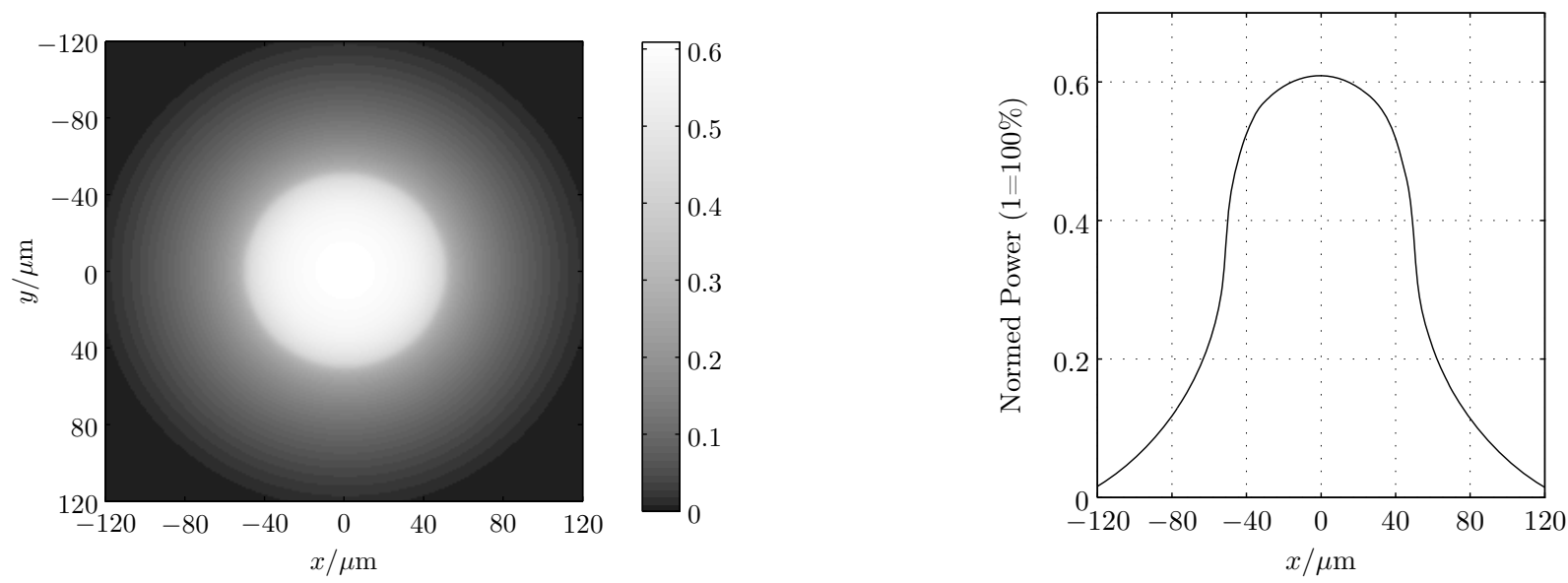

Figure 9. Power Distribution for one fiber as function of the spot position corresponding to the fiber's center for a distance behind the focusing lens of $296.5 \mathrm{~mm}$

Left: Full Distribution, Right: Cut through Full Distribution for $y=0$

\subsection{Simulation Results}

For the execution of the simulation, the position of the spots described in Section 4.2 was varied over the whole Fiberbundle's surface. For each point the powers in the fibers were determined and a position calculation by means of Equations 1 and 2 has been done. This procedure results in a 3-dimensional distribution of the calculated position over the given position and is shown in Figure 10.
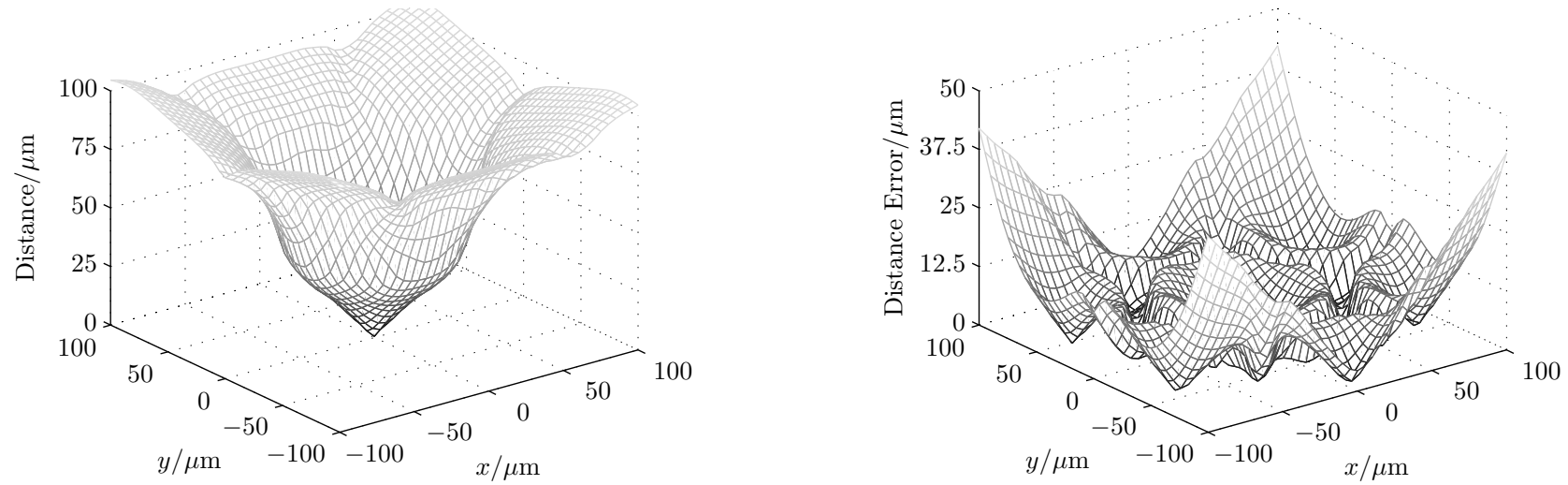

Figure 10. Simulation Results for a distance behind the focusing lens of $296.5 \mathrm{~mm}$

Left: Calculated Position vs. Actual Position, Right: Position Error vs. Actual Position

Since such diagrams are not suitable to draw conclusions, only cuts through this distributions for $y=0$ are shown in following diagrams. This neglects the fact that the distributions are not fully rotation-symmetric, but the tendency of the 2-dimensional graphs is the same and therefore the results can be used for evaluations.

These 2-dimensional graphs are shown in Figure 11 for different distances. The left hand side shows the calculated position over the given position. On the right hand side, the error of the calculations over the given position is displayed.

A highly unsteady behaviour can be observed for small spot diameters, as it is the case for a distance of $296.0 \mathrm{~mm}$. This is justified by the fact that no control signal can be generated, because the full spot power is present in the central fiber for a wide range of spot offsets from the center.

Due to the fact that the side lobes of the spots reach out further for greater distances (cp. Figure 8), the results get more reasonable for this cases. The result is a good control signal even for small offsets from the center. 

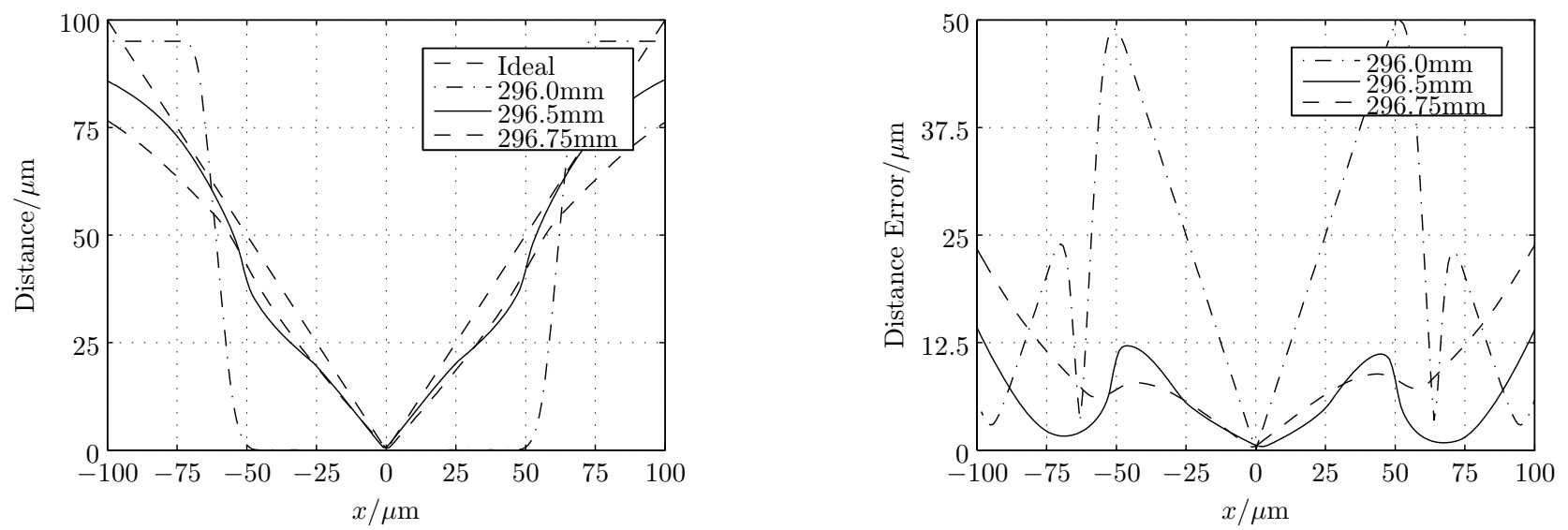

Figure 11. Simulation Results for different distances, cut through Full Distributions for $y=0$

Left: Calculated Position vs. Actual Position, Right: Distance Error vs. Actual Position

It is remarkable that, although the position calculation does not supply an accurate result, a linear characteristic is present for small position offsets $(x<25 \mu \mathrm{m})$. Hence the deficit in accuracy can be overcome by adjusting the proportional gain parameter of the controller, and does therewith not have a negative influence on the system performance.

For the further investigations that are completed in this paper, a distance of $296.5 \mathrm{~mm}$ has been chosen. The power loss with an FWHM-diameter of $75.5 \mu \mathrm{m}$ is still reasonable, while a good behaviour of the controller can be expected.

\section{VALIDATION TEST RESULTS}

\subsection{Overview}

The parameters of the PID-Controller were optimized in a lab environment by using the well-known ZieglerNichols-Method. The so-found parameters were set up in the controller and used for the following measurements.

Two measurements were accomplished for the evaluation of the tracking behaviour: First, a jump response of the system was recorded. Secondly, the system was installed in a vibrating environment, in order to test it under real operation conditions. It would have been desirable to run an exhaustive test on e.g. a vibrating table, but no such device was accessible at the time when the tests were executed.

\subsection{Jump Response}

For the purpose of evaluating the performance of the control loop, the jump response of the system was recorded. Therefore the mirror was adjusted in such a way that the impinging power was solely focused on Fiber 2 (cp. Figure 4), while the control algorithm was turned off. Then the sending laser was switched off and the controller was enabled. After switching the sending laser back on, the spot was steered back to the central fiber by the controller. An oscilloscope was used to record the signals displayed in Table $3^{*}$. The result of this measurement is shown in Figure 12.

\begin{tabular}{cc}
\hline Signal & Description \\
\hline$P_{2} / W$ & Power at photodetector 2 \\
$P_{2}^{\prime} / W$ & Power after anti-aliasing lowpass \\
$U_{M y} / V$ & Control signal for y-axis of mirror \\
$U_{M \text { y pos }} / V$ & Mirror position \\
\hline
\end{tabular}

Table 3. Recorded Data for the Jump-Response Measurement

\footnotetext{
${ }^{*}$ Voltages were converted to optical powers, where appropriate
} 

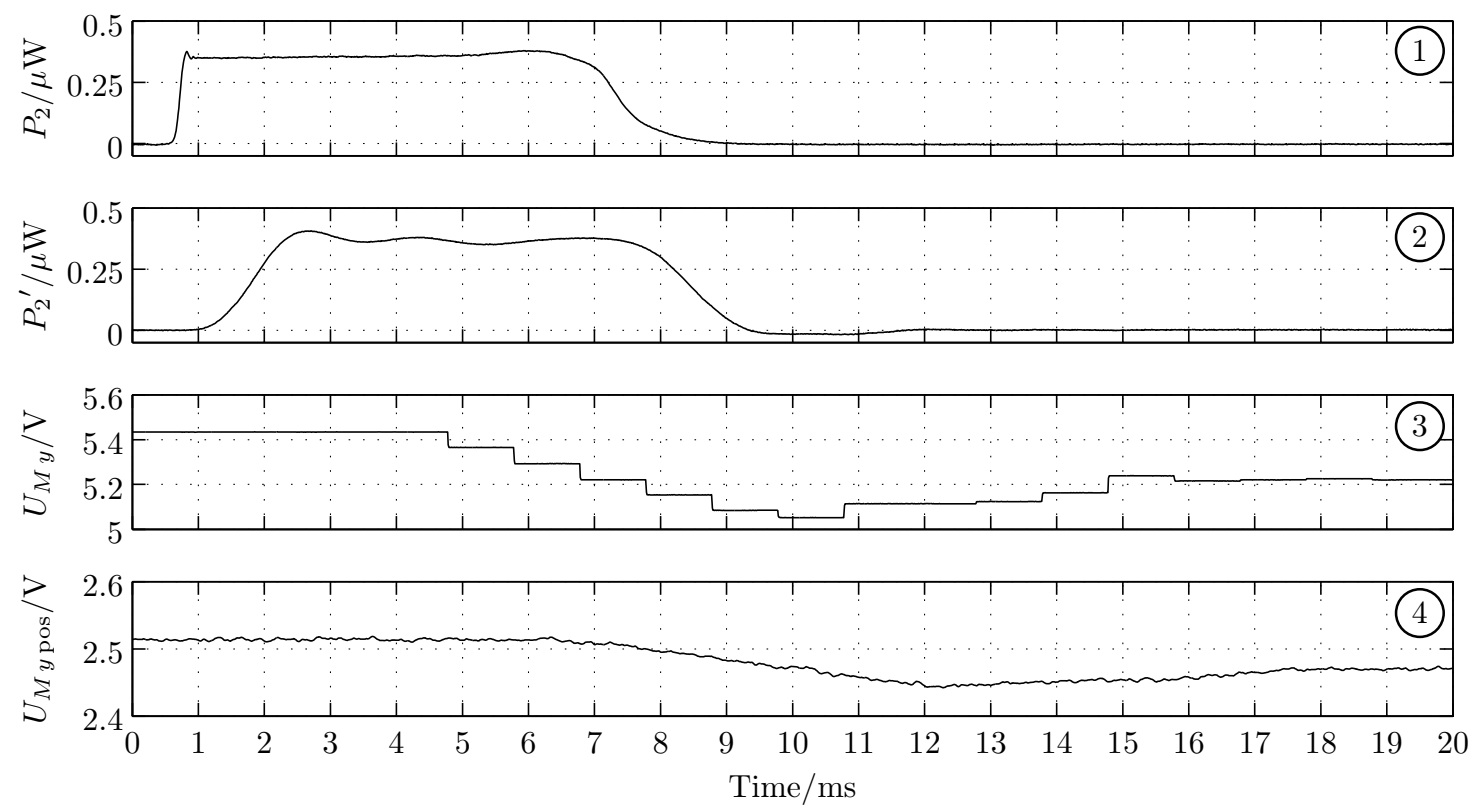

Figure 12. Jump Response of the System (cp. Table 3)

Graph (1) shows the power in Fiber 2 over the measurement time. It can be observed that the anti-aliasing lowpass introduces a small signal delay (2). The time until the controller reacts and starts to adjust the control voltage of the mirror system is around $5 \mathrm{~ms}(3)$. The disturbance is fully compensated after a time of around $18 \mathrm{~ms}$, as can be seen in the position signal of the mirror system (4). A slight overshoot of the controller occurs, what implies that the PID-parameters are well optimized.

The reaction time of the system can be found to be in the range of a few ms. Due to the fact that the utilized fast steering mirror is capable of fulfilling movements at higher speeds, a significant performance improve can be expected for a reduced reaction time, as it would be the case for a faster control algorithm with a higher sampling rate.

\subsection{Vibrating Environment}

The system has been set up on the institute's measurement van, in order to provide a vibrating environment for further testing. During the measurements, the engine of the bus remained running and thus the system was set under vibrations. The temperatures at the day of the measurement were around $0^{\circ} \mathrm{C}$, while slight snow showers occurred. The link distance was about $10 \mathrm{~m}$.

Figure 13 shows the power in the central fiber over time for the measurement, both with disabled (left graph) and enabled (right graph) controller. The sampling rate was $1 \mathrm{kHz}$. It is visible that the power was continuously impinging in the central fiber for the case with enabled controller. For the case without controller, slow power fluctuations due to wind and other environmental influences moving the van can be observed.

In order to evaluate the system performance with more detail, the spectra of the time functions were calculated and are given in Figure 14. A suppression of low frequencies is clearly visible in the case with enabled controller. Also the peaks between $10 \mathrm{~Hz}$ and $100 \mathrm{~Hz}$, that were caused by the van's engine, turn out to be smaller.

However, the influence of the tracking system is rather small for frequencies above $5 \mathrm{~Hz}$. Due to the fact that the actual vibration conditions of the van are not known with high accuracy, and that the sources of the high-frequency noise visible in the spectra are unclear, no conclusions about the system performance can be drawn here. 

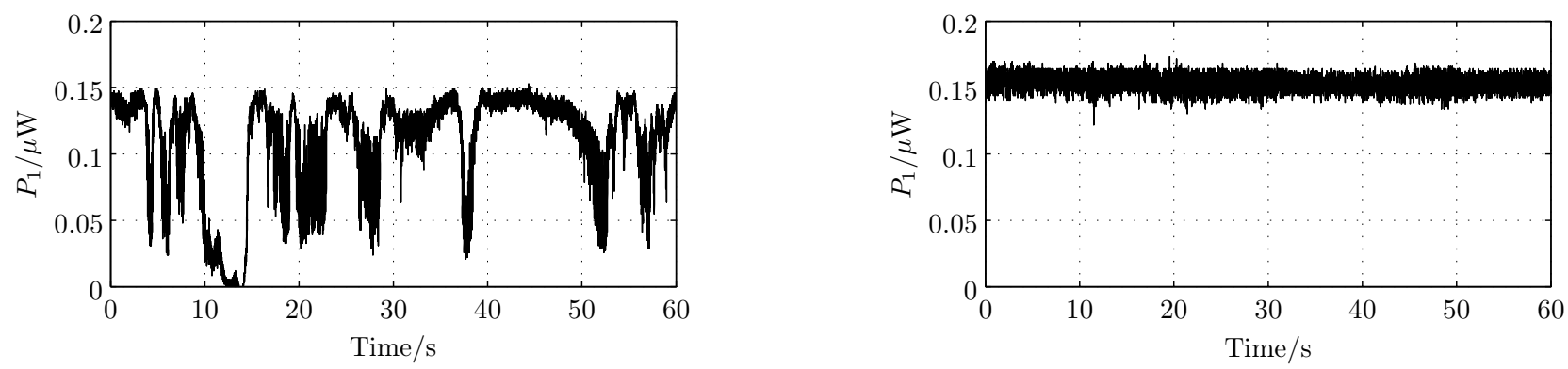

Figure 13. Power over time in Fiber 1 for the Fiberbundle Receiver in a Vibrating Environment Left Graph: Controller disabled, Right Graph: Controller enabled
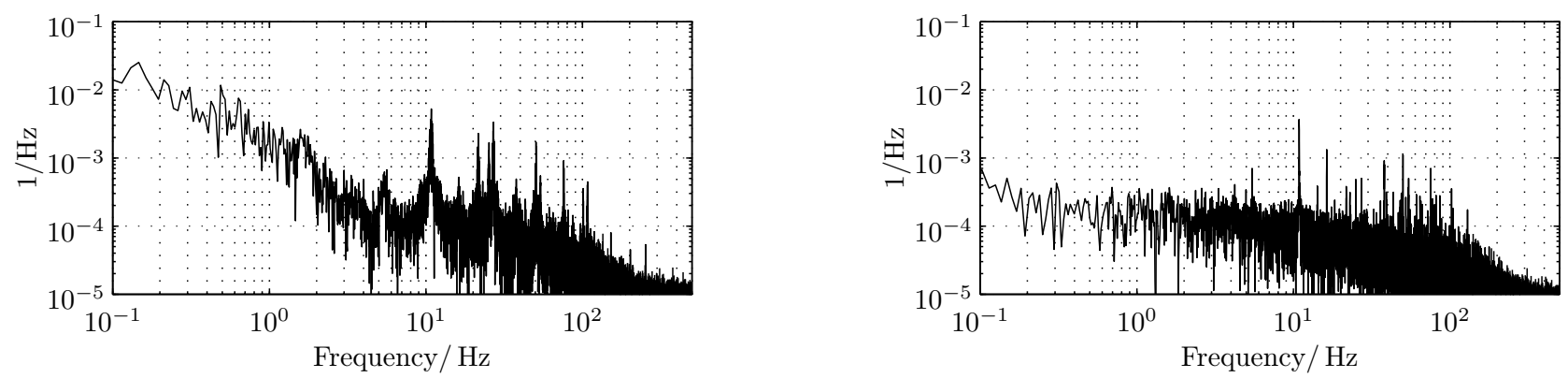

Figure 14. Spectrum of the Power in Fiber 1 for the Fiberbundle Receiver in a Vibrating Environment

Left spectrum: Controller disabled, Right spectrum: Controller enabled

\section{CONCLUSIONS}

It has been shown that a Fiberbundle can be used as cost-effective and fast tracking device for transceivers in mobile free space optical communication systems. Its application simplifies the implementation process of optical transceivers.

However, the experimental system described in this paper doesn't fully exploit the achievable performances of a Fiberbundle system. It turned out that the sampling rate of $1 \mathrm{kHz}$ is not fast enough in order to obtain an agile system, that makes full use of the capabilities of the utilized fast steering mirror. Therefore the sampling rate of the system should be increased.

Also the effect of multimode-dispersion and its influence on the achievable bandwidth in this specific scenario should be evaluated with more detail. If the outcome is that the datarate limit is too restricting for practical applications, another Fiberbundle with smaller fibers (e.g. with a core-diameter of $62.5 \mu \mathrm{m}$ and thus with a higher Bandwidth-Length-Product) could be used.

For the purpose of determining the system performance, more sophisticated devices such as a vibrating table are necessary. The approach to measure the system performance by mounting it on a van showed the principal operability, but was not suitable for detailed evaluations.

Further improvements include the usage of avalanche- instead of PIN-photodiodes to increase the system's sensitivity. On the other hand, this introduces more complexity due to the fact that the multiplication-factor of APDs is very sensitive to temperature changes and thus makes comparative power measurements complicated.

In applications where a high Field of View is required, it could be enlarged by using a greater Fiberbundle with a second ring of fibers. These could have larger diameters, what would enable their use for acquisition purposes.

\section{REFERENCES}

1. F. David, D. Giggenbach, R. Landrock, J. Horwath, K. Pribil, E. Fischer, R. Buschner, and D. Blaschke, "Overview of the FASOLT Experiment and Final Results," in LASE 2003, San Jose, California, USA, 25th31st January 2003, Proceedings of the SPIE, Vol. 4975, 2003. 
2. J. Horwath, M. Knapek, B. Epple, M. Brechtelsbauer, and B. Wilkerson, "Broadband Backhaul Communication for Stratospheric Platforms: The Stratospheric Optical Payload Experiment (STROPEX)," in Free-Space Laser Communications VI, Proceedings of SPIE, Vol. 6304, 2006.

3. N. Perlot, M. Knapek, D. Giggenbach, J. Horwath, M. Brechtelsbauer, Y. Takayama, and T. Jono, "Results of the Optical Downlink Experiment KIODO from OICETS Satellite to Optical Ground Station Oberpfaffenhofen (OGS-OP)," in Conference on Laser Communication and Propagation, Proceedings of the SPIE, Vol. $6457 \mathrm{~A}, 2007$.

4. H. Henniger and D. Giggenbach, "Avionic Optical Links for High Data-Rate Communications," in 25th Congress of International Council of the Aeronautical Sciences, Proceedings of ICAS - 25th Congress of the International Council of the Aeronautical Sciences , 2006.

5. C. Fuchs, H. Henniger, G. Di Nepi, and et al., "Broadband Communications for Aeronautical Networks: The ATENAA Outer Optical Link Validation," in 1st CEAS Air and Space Conference, Berlin, Germany, 10th-13th September 200\%, 2007.

6. C. Fuchs, "Entwicklung eines Faserbündelempfängers für die optische Freiraumkommunikation," Master Thesis, German Aerospace Center (DLR), Oberpfaffenhofen, Germany, 2006. 